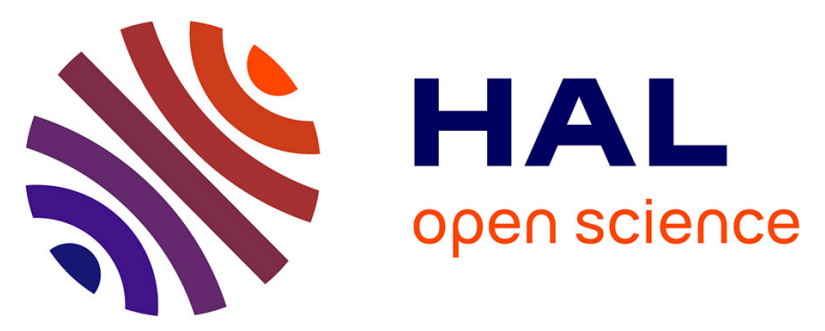

\title{
Single-Phase Wireless Power Transfer System Controlled by Magnetic Core Reactors at Transmitter and Receiver
}

Luis Romba, Stanimir S. Valtchev, Rui Melício

\section{To cite this version:}

Luis Romba, Stanimir S. Valtchev, Rui Melício. Single-Phase Wireless Power Transfer System Controlled by Magnetic Core Reactors at Transmitter and Receiver. 8th Doctoral Conference on Computing, Electrical and Industrial Systems (DoCEIS), May 2017, Costa de Caparica, Portugal. pp.419-428, 10.1007/978-3-319-56077-9_41. hal-01629574

\section{HAL Id: hal-01629574 \\ https://hal.inria.fr/hal-01629574}

Submitted on 6 Nov 2017

HAL is a multi-disciplinary open access archive for the deposit and dissemination of scientific research documents, whether they are published or not. The documents may come from teaching and research institutions in France or abroad, or from public or private research centers.
L'archive ouverte pluridisciplinaire HAL, est destinée au dépôt et à la diffusion de documents scientifiques de niveau recherche, publiés ou non, émanant des établissements d'enseignement et de recherche français ou étrangers, des laboratoires publics ou privés. 


\title{
Single-phase Wireless Power Transfer System Controlled by Magnetic Core Reactors at Transmitter and Receiver
}

\author{
Luis Romba ${ }^{1}$, Stanimir S. Valtchev ${ }^{1}$ and Rui Melício ${ }^{2,3}$ \\ ${ }^{1}$ UNINOVA-CTS and Faculdade de Ciências e Tecnologia, \\ Universidade Nova de Lisboa, Portugal \\ ${ }^{2}$ IDMEC, Instituto Superior Técnico, Universidade de Lisboa, Portugal \\ ${ }^{3}$ Departamento de Física, Escola de Ciências e Tecnologia, Universidade de Évora, Portugal, \\ luis.rjorge@netvisao.pt,ssv@fct.unl.pt, ruimelicio@gmail.com
}

\begin{abstract}
The applications of wireless power transmission have become widely increasing over the last decade, mainly in the battery charging systems for electric vehicles. This paper focuses on the single-phase wireless power transfer prototype controlled by magnetic core reactors in either side of the system: that of the transmitter, and that of the receiver. The described wireless power transfer system prototype employs a strong magnetic coupling technology to improve the power transmission efficiency. In the same time, a magnetic core reactor is used to control the "tuning" between the transmitter and the receiver frequencies, allowing for that increase of the system efficiency. Finally, practical results of the implemented prototype are presented.
\end{abstract}

Keywords: Wireless power transfer, strongly magnetic coupling, magnetic core reactor.

\section{Introduction}

The Wireless Power Transfer (WPT) is a system that allows the transfer of electric power from a point in space to another point in space without any physical support, i.e., without wires or galvanic contact [1]. For relatively short and midrange distances, i.e., from a few millimeters to some meters, the transmission is performed mainly by magnetic field. The WPT systems used at short distances have a wide range of purposes. The applications start from battery charging of domestic appliances, mobile phones and laptops, even of Implantable Medical Devices (IMD), e.g. pacemakers $[2,3]$. The performance of these devices is largely dependent on the high efficiency of the power transfer. The best way to optimize the power transfer efficiency is to increase the (magnetic) coupling between the two coils. This is one of the rules of thumb that should be applied to the WPT systems [4].

Due to the increased demand for the green energy systems, the WPT systems for midrange distances have had a significant development in the research related to battery charging applications for hybrid and electric vehicles. Hence, in the practice the midrange battery charger uses the strongly coupled magnetic resonance technology [5]. The strongly coupled magnetic resonance technology is characterized 
by two pairs of control loops, one for the transmitter the other for the receiver (Fig. 1). The internal impedance of the Source Generator can be annotated later as $R_{s}$, and the impedance of the Load can be marked as further on as $R_{l}$. Those two impedances (real, at the resonant frequency) are excluded further on, from the $R L C$ resonators, leaving the resonator circuits independent of the generator and the load.

The equivalent circuit of the WPT using strongly coupled magnetic resonance technology is shown in Fig. 1.
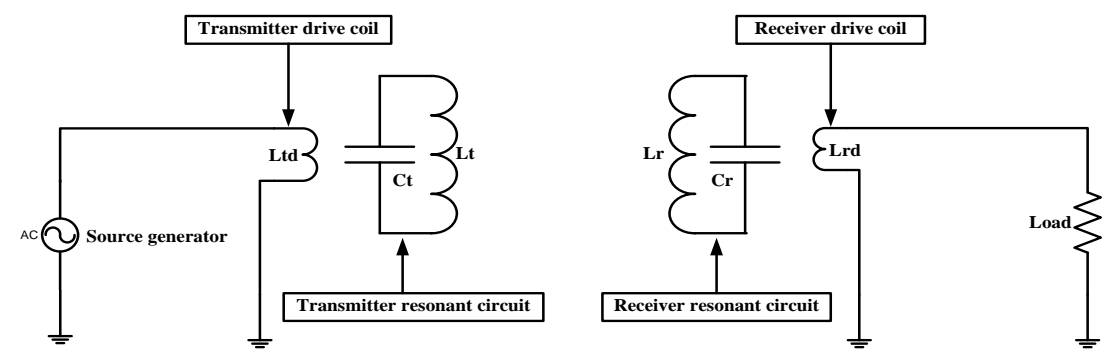

Fig.1. Simplified equivalent circuit of the WPT system.

The transmission efficiency, when a strongly coupled magnetic resonance technology is used, drops down with any directional misalignment. The maximum efficiency occurs when a correct impedance matching between the source and load will be achieved [6].

The impedance adjustment can be obtained in two ways. One way is by tuning the operating frequency, and the other is by keeping a fixed operating frequency but tuning the impedance matching between the transmitter and the receiver systems $[6,7]$. The described WPT system includes two magnetic core reactors (MCR), one on each side of the system, i.e., the transmitter and the receiver, as shown in Fig. 2.

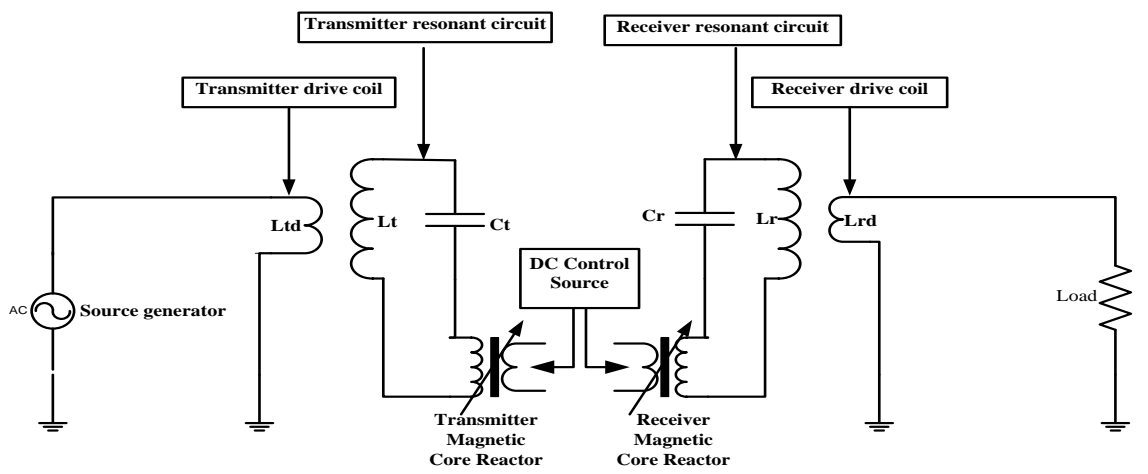

Fig. 2. WPT with magnetic core reactor in both sides. 
The MCR is in fact, a part of the well-known in the past "magnetic amplifier" [8]. It is formed by two circuits, one is the control or primary circuit where a DC current is applied for controlling the secondary circuit, where the circulation of $\mathrm{AC}$ current is regulated [8]. When the control current (DC) changes, it causes a variation of the magnetic flux. This change in the magnetic flux produces a variation of the magnetic permeability of the circuit. If the circuit is working in the linear part of the curve of the hysteresis cycle, the variation of the magnetic flux is approximately constant [9]. However, if the operating point is above the knee of the curve of the hysteresis loop, the variation is always much more significant and the control is more efficient. This enables the frequency adjustment to be carried out either at the transmitter or the receiver of the WPT system, by changing the value of the inductance connected in series with the corresponding resonant coil, causing a change in the total circuit inductance $[8,9]$.

\section{Relationship to Smart Systems}

In contemporary societies the combination of nanoelectronics, embedded systems, cybernetics and intelligent systems, has and will continue to have a fundamental importance. That combination (smart system) is present in almost all modern products and services [10], being the decisive set of elements and key enabling technologies in many innovations and solutions for societal challenges, being also a very significant factor in the competitiveness, employment and prosperity of countries and regions [10].

The smart systems combine cognitive functions with sensing, actuation, data communication and energy management in an integrated way. The enabling principles of this combination are now the nanoelectronics, micro-electro-mechanics, magnetism, photonics, chemistry and radiation [11]. The smart systems are integrated with the (natural, built and social) environment, networks for power and data, other smart systems and the human beings [11].

The mobility sector is facing major challenges, e.g. the $\mathrm{CO}_{2}$ emissions reduction, together with the implied improvement of air quality especially in large cities and the reduction of traffic congestion still using the existing infrastructure wherever possible.

The development is made possible by electronics, with new components and systems, as well as by introduction of new methods and tools. It has come to support the concepts that will provide new vehicles, transport systems and infrastructures with intelligence and flexibility to meet the challenges of industry in this sector [12]. The innovation, development and research related to the smart mobility has its emphasis on the capabilities in the areas of detection, communication, geo-positioning, computing and decision making and control based on Electronics, Components and Systems (ECS) [12]. ECS functions will lead to resource-efficient transportation as they enable partly or fully electrified as well as advanced conventional vehicles that are clean, $\mathrm{CO}_{2}$-optimized and smartly connected to renewable energy sources [12].

Two examples of the development of specific applications in smart mobility are: smart systems for electro-mobility and smart systems for automated driving. In the first are included electric drive train, battery and battery management and charging 
technologies and the second is the included automated driving without the driver in the loop, on highways and in urban regions, but allowing the driver to intervene [11].

\section{Modelling}

The magnetic core circuit of a WPT system, when a correct alignment is achieved, is shown in Fig. 3. The coils are wound on the middle leg of each "E" part of the cores, upper "E" part corresponding to the primary, and the bottom part to the secondary. According to the structure of the magnetic core and the dimension of the air gap, the reluctance values are indicated as follows: $\Re_{1}, R_{2}, \Re_{2} \Re_{4}, R_{5}$ for the primary branches, and $\Re_{6}, \Re_{7}, \Re_{g} \Re_{9}, \Re_{10}$ are the secondary parts of the magnetic core reluctance. The $\Re_{a g 1}, \Re_{a g 22}, R_{a g a}$, are the reluctance elements that correspond to the

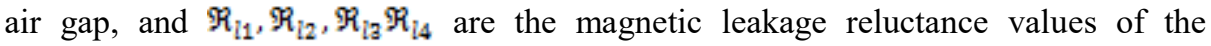
primary and the secondary. The simplified equivalent magnetic circuit is shown in Fig. 4, where seven loops or sub-circuits are marked.

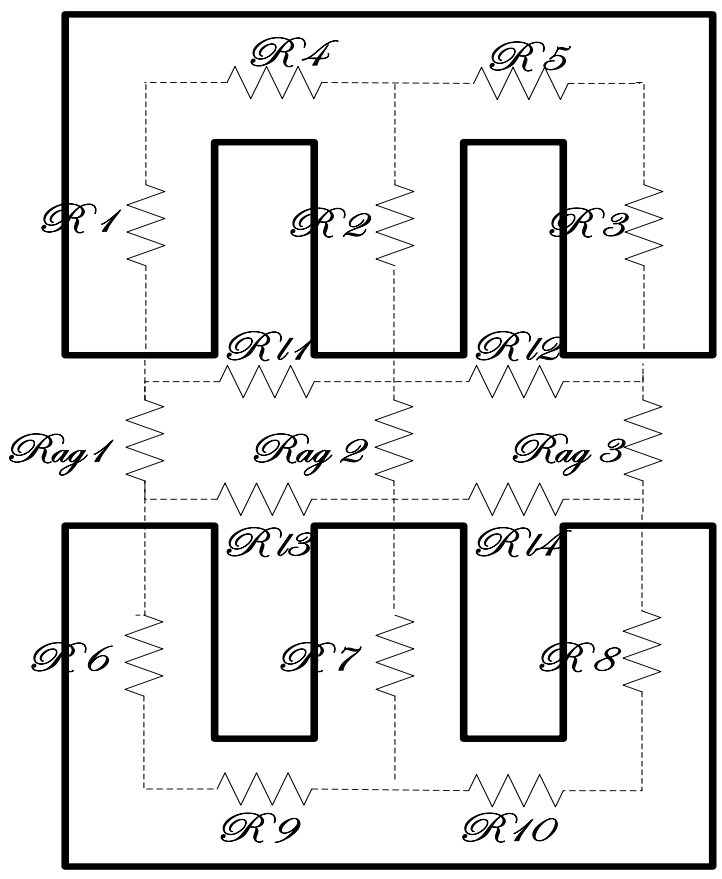

Fig. 3. Simplified equivalent magnetic circuit.

Based on this circuit (Fig. 3), the equation of the total reluctance can be deduced as: 
$R_{\text {Total }}$
$=2 \frac{\ell_{c}}{\mu_{0} \mu_{Y} S_{c}}$
$+\frac{3\left(\frac{\ell_{a g}}{\mu_{0} S_{a g}}\right)\left(\frac{\ell_{\ell}}{\mu_{0} S_{\ell}}\right)^{2}+12\left(\frac{\ell_{\ell}}{\mu_{0} S_{\ell}}\right)\left(\frac{\ell_{a g}}{\mu_{0} S_{a g}}\right)\left(\frac{\ell_{c}}{\mu_{0} \mu_{r} S_{c}}\right)+4\left(\frac{\ell_{c}}{\mu_{0} \mu_{r} S_{c}}\right)\left(\frac{\ell_{\ell}}{\mu_{0} S_{\ell}}\right)^{2}}{2\left(\frac{\ell_{\ell}}{\mu_{0} S_{\ell}}\right)^{2}+16\left(\frac{\ell_{\ell}}{\mu_{0} S_{\ell}}\right)\left(\frac{\ell_{c}}{\mu_{0} \mu_{r} S_{c}}\right)+6\left(\frac{\ell_{\ell}}{\mu_{0} S_{\ell}}\right)\left(\frac{\ell_{a g}}{\mu_{0} S_{a g}}\right)+24\left(\frac{\ell_{c}}{\mu_{0} \mu_{r} S_{c}}\right)\left(\frac{\ell_{a g}}{\mu_{0} S_{a g}}\right)}$

The result is shown in Fig. 4.

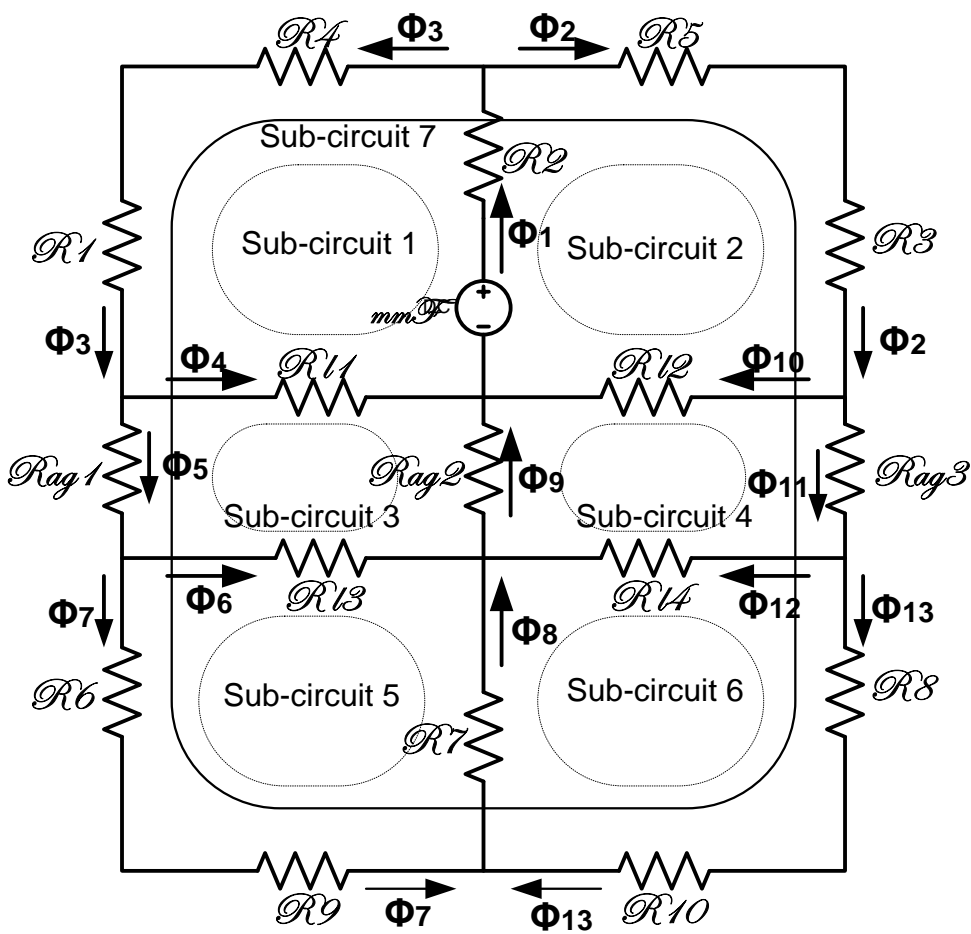

Fig. 4. Equivalent magnetic circuit with sub-circuits.

The variation of $\Re_{\text {Total }}$, as function of $\ell_{a g}$, fixed all the others parameters is shown in Fig. 5. 


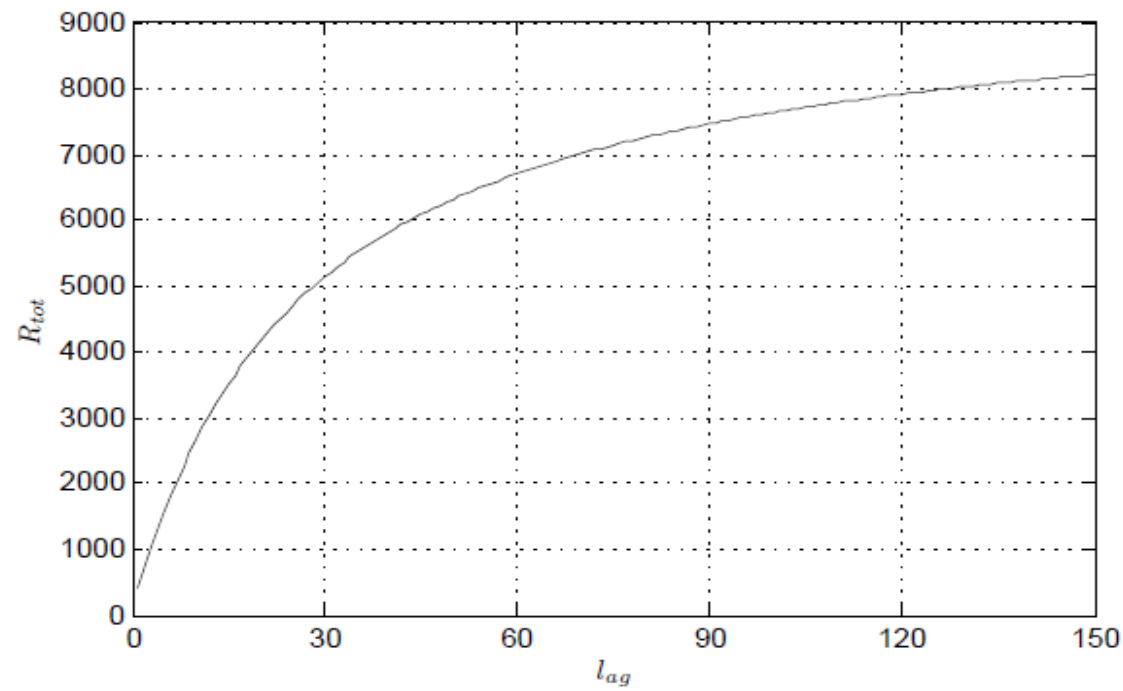

Fig. 5. Variation of total magnetic reluctance function of the air gap.

The mutual inductance, $M_{p s}=M_{s p}=M$ is given by:

$$
\mathrm{M}=\frac{\mathrm{N}_{\mathrm{p}^{*}} \mathrm{~N}_{s}}{\Re_{\text {Total }}}
$$

The result of the replacing of the (1) in (2) is given by:

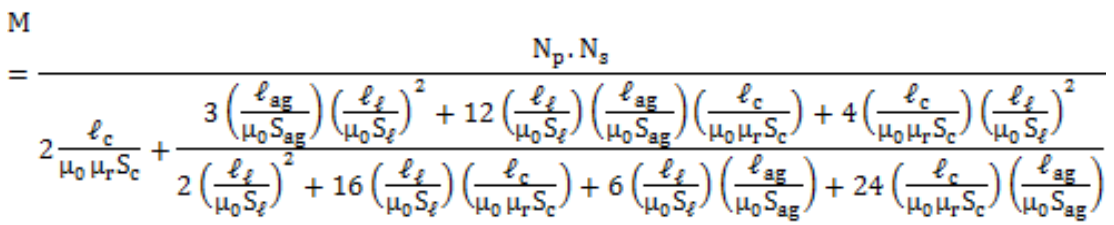

The coupling coefficient is given by:

$$
k=\frac{M}{\sqrt{L_{p^{*}} L_{s}}}
$$

Replacing (3) into (4) results in: 


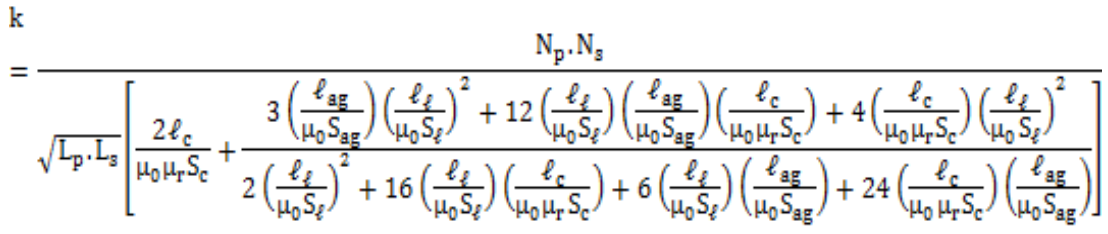

\section{Case Study}

The global physical model presented in this paper was performed in Power Electronics Laboratory, Faculty of Science and Technology, New University of Lisbon and is shown in Fig. 6. The implemented schematic diagram is based on Fig. 2.

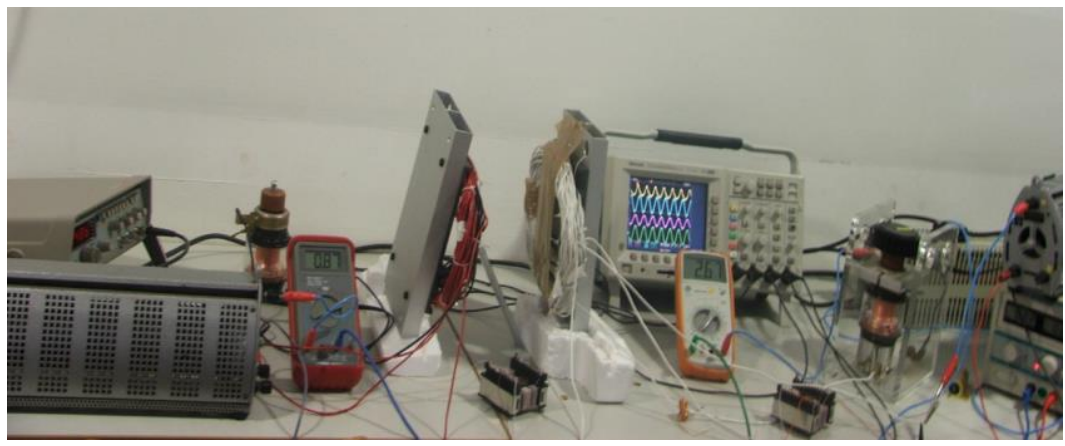

Fig. 6. Global physical model implemented.

The transmitter coils set, the receiver coils set and the two magnetic core reactors, one for each circuit $[13,14]$ are shown in Fig. 7.

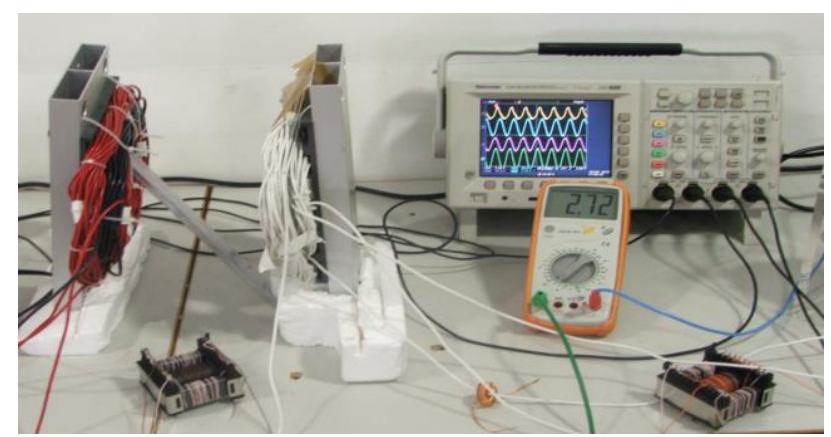

Fig. 7. Partial view implemented circuit with the two MCRs. 
The tests were conducted step by step. In the first step a frequency of the function generator was selected (Fig. 2). This choice is a hardware simulation of the reality where the transmitter frequency is defined as the transmitter control has decided. The second step was to tune the transmitter resonant circuit by applying DC current to the transmitter magnetic core reactor until obtaining the maximum voltage that is observed at the resonant circuit. This step corresponds to the set point optimization. The third step was to tune the receiver resonant circuit following an identical procedure to the previous step, i.e. obtaining the maximum voltage at the receiver resonant circuit. During those procedures, a constant load value was maintained. The values of the total inductance for the transmitter and for the receiver are shown in Fig. 8 .

The resonant frequency values for each inductance value, maintaining constant the capacitance, are shown in Fig. 9.

The measured voltages by the oscilloscope (Fig. 10) show the input voltage (channel 1), and the voltage value in the transmitter resonant circuit (channel 2). The channel 3 is the voltage value measured at the receiver resonant circuit. The channel 4 is the voltage value applied to the load.
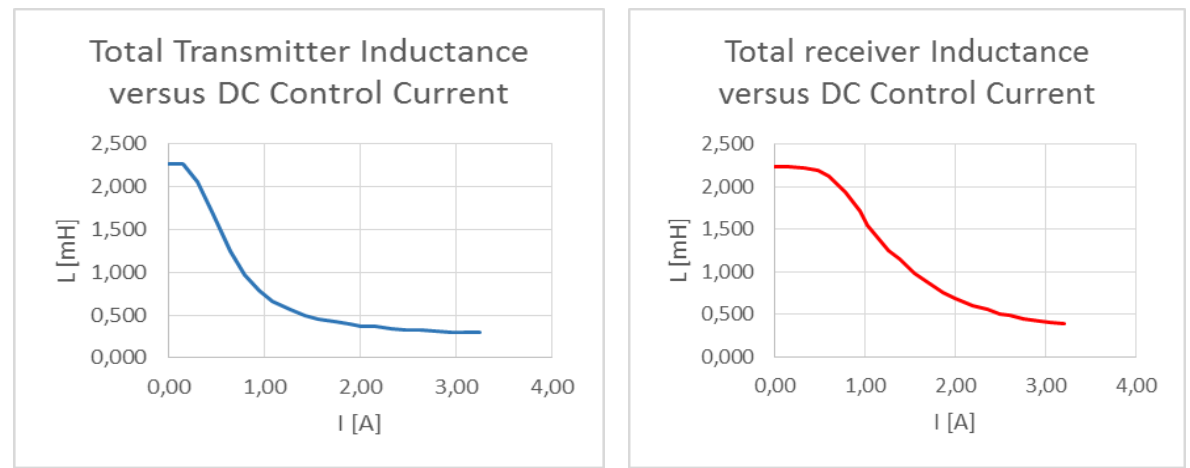

Fig. 8. The total Inductance values to the transmitter and receiver.

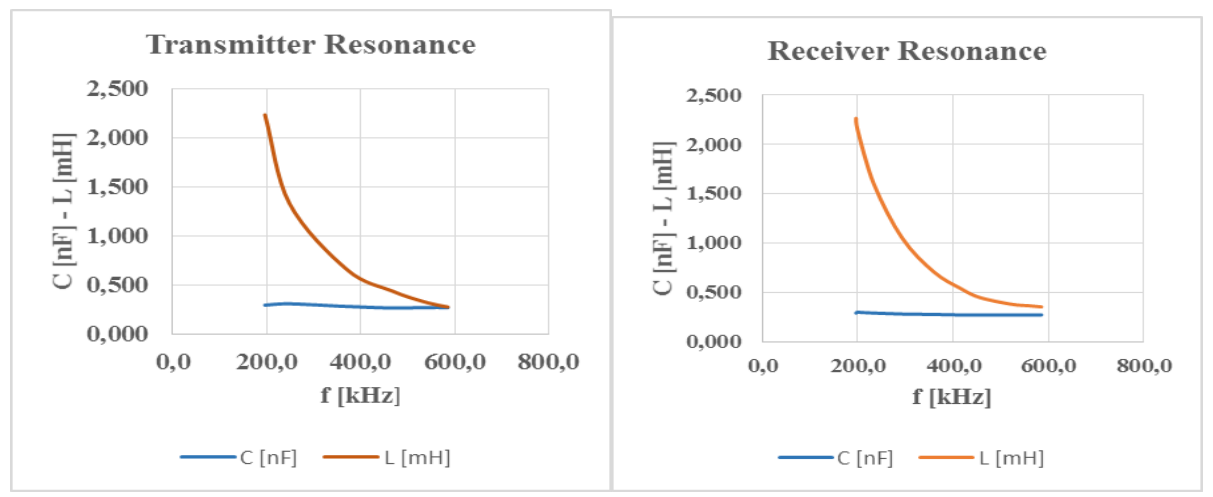

Fig. 9. The resonance values to the transmitter and the receiver. 
The voltage values in each test point of the circuit (channels 1, 2, 3, 4) are shown in Fig. 10.

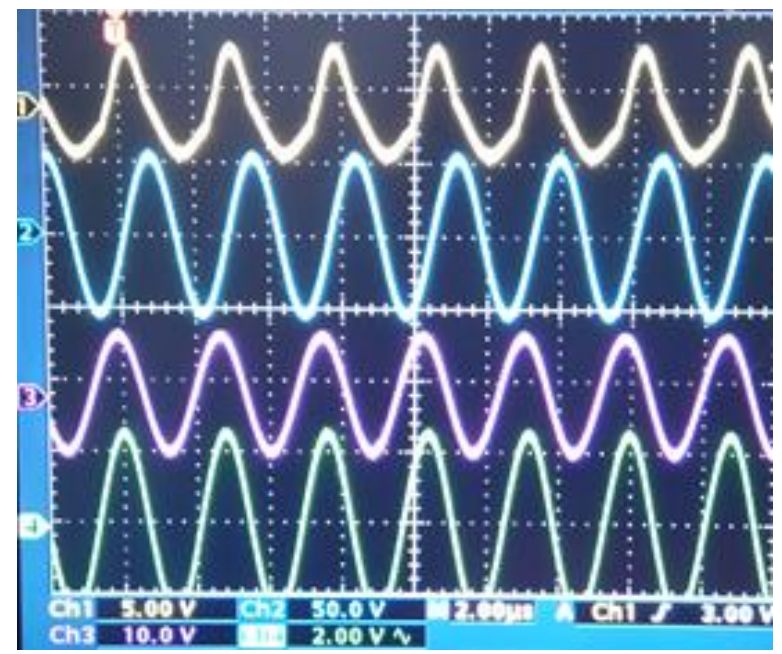

Fig. 10. The voltage values in each test point of the circuit (channels 1, 2, 3, 4).

\section{Conclusions}

In this paper a different approach is presented, applying the magnetic reluctance elements to determine the mutual inductance and the coupling coefficient. This process makes possible to obtain, with reasonable precision, the most important values in order to design a project of a wireless power transfer through a simple distances measurement. In the case study, it is shown a WPT implemented physical model in which a frequency control is presented using two magnetic core reactors. Through the curves shown in Fig. 8 and in Fig. 9, it is possible to predict the DC current that will be necessary for controlling the magnetic core reactors, maintaining constant the resonant capacitor values, and in the same time, to tune the transmitter and the receiver at the same frequency. The stronger magnetic coupling, as presented in the circuits, shown in the Fig. 6 and in Fig. 7, allows to obtain a sine wave at the output even if the input signal is not a pure sinusoidal wave. As it is shown in Fig. 10, it is made possible by the two resonant circuits mounted at each side: the transmitter (distorted, channel1) and the receiver (channel 4).

\section{References}

1. Ke, L., Yan, G., Yan, S., Wang, Z., Liu, D.: Improvement of the coupling factor of Litz-wire coil pair with ferrite substrate for transcutaneous energy transfer system. Progress in Electromagnetics Research, 39, 41-52 (2014) 
2. Park, S.I.: Enhancement of wireless power transmission into biological tissues using a high surface impedance ground plane. Progress in Elec. Research, 135, 123-136 (2013)

3. Jolani, F., Mehta, J., Yu, Y., Chen, Z.: Design of wireless power transfer systems using magnetic resonance coupling for implantable medical devices. Progress in Electromagnetics Research Letters. 40, 141-151 (2013)

4. Benjamin, L.C., Hoburg, J.F., Stancil, D.D., Goldstein, S.C.: Magnetic resonant coupling as a potential means for wireless power transfer to multiple small receivers. IEEE Transactions on Power Electronics. 24(7), 1819-1825 (2009)

5. Qiu, C., Chau, K.T., Ching, T.W., Liu, C.: Overview of wireless charging technologies for electric vehicles. Journal of Asian Electric Vehicles, 12(1), 1679-1685 (2014)

8. Xu, D.Q., Joós, G., Lévesque, M., Maier, M.: Integrated V2G, G2V, and renewable energy sources coordination over a converged fiber-wireless broadband access network. IEEE Transactions on Smart Grid, 4(3), 1381-1390 (2013)

9. Fabbri, G., Tarquini, G., Pasquali, L., Anniballi, L., Odoardi, S., Teodori, S., Santini, E.: Impact of $\mathrm{V} 2 \mathrm{G} / \mathrm{G} 2 \mathrm{~V}$ technologies on distributed generation systems. In: 23th IEEE Int. Symp. on Industrial Electronics, 1-4, Istanbul, Turkey (2014)

6. Austrin, L., Engdahl, G.: Modeling of a three-phase application of a magnetic amplifier. In: 24th International Congress of the Aeronautical Sciences, 1-8, Yokohama, Japan (2004)

7. Trinkaus, G.: Magnetic amplifiers another lost technology. Based on original work from U.S. Navy 1951, High Voltage Press, USA (2000)

10.Thales, L.G.: ARTEMIS-IA Pre-Brokerage event, Brussels, Belgium (2014)

11.Neul, N.: Smart Systems Integration in the Context of ECSEL, Munich, Germany (2016)

12.Multi Annual Strategic Research and Innovation Agenda for ECSEL Joint Undertaking (2016)

13.Romba, L.F.R., Valtchev, S.S., Melício, R.: Improving magnetic coupling for battery charging through 3D magnetic flux. In: IEEE International Power Electronics and Motion Control Conference, 291-297, Varna, Bulgaria (2016)

14.Romba, L.F.R., Valtchev, S.S., Melício, R.: Three-phase magnetic field system for wireless energy transfer. In: IEEE International Symposium on Power Electronics, Electrical Drives and Motion, 73-78, Capri, Italy (2016) 